\title{
Odds ratio: aspectos teóricos y prácticos
}

\author{
JAIME CERDA ${ }^{1,2}$, CLAUDIO VERA $^{1,3}$, GABRIEL RADA $^{1,4}$
}

\section{Odds ratio: Theoretical and practical issues}

Odds Ratio (OR) is an effect measure frequently used to communicate results of health research. Mathematically, OR is the quotient between two odds, being odds an alternative way to express possibility of occurrence of an outcome or presence of an exposition. From a methodological perspective, $\mathrm{OR}$ can be calculated from prospective, retrospective and cross-sectional designs, and under certain conditions it can replace the Relative Risk. Based on a series of questions and examples, this article explains theoretical and methodological grounds underlying the concept of $O R$, in order to facilitate its interpretation for clinicians and researchers.

(Rev Med Chile 2013; 141: 1329-1335).

Key words: Biostatistics; Odds Ratio; Research report.

\section{Resumen}

Odds Ratio (OR) es una medida de efecto comúnmente utilizada para comunicar los resultados de una investigación en salud. Matemáticamente un OR corresponde a un cociente entre dos odds, siendo un odds una forma alternativa de expresar la posibilidad de ocurrencia de un evento de interés o de presencia de una exposición. Desde un punto de vista metodológico, los OR pueden ser calculados en diseños prospectivos, retrospectivos y transversales, y bajo ciertas condiciones pueden reemplazar al Riesgo Relativo. En base a una serie de preguntas y ejemplos, el presente artículo explica los fundamentos matemáticos y metodológicos subyacentes al concepto de $O R$, con el objetivo de facilitar su interpretación a clinicos e investigadores.
'Unidad de Medicina Basada en Evidencia. ${ }^{2}$ Departamento de Salud Pública.

${ }^{3}$ División de Obstetricia y

Ginecología.

${ }^{4}$ Departamento de Medicina Interna. Facultad de Medicina, Pontificia Universidad Católica de Chile. Santiago de Chile.

Los autores declararon no tener conflictos de intereses en este manuscrito.

Recibido el 4 de enero de 2013, aceptado el 2 de mayo de 2013.

Correspondencia a:
Dr. Jaime Cerda.
Departamento de Salud
Pública, Facultad de
Medicina, Pontificia
Universidad Católica de
Chile. Marcoleta 434,
Piso 1. Santiago Centro.
Teléfono: (56-2) 354-3038
E-mail: jcerda@med.puc.cl

$\mathrm{R}$ azón de productos cruzados, razón de chances, razón de ventajas, razón de momios, desigualdad relativa y oportunidad relativa son algunos de los nombres propuestos para traducir el término inglés Odds Ratio (OR). Esta medida de efecto es comúnmente utilizada para comunicar los resultados de una investigación en salud ${ }^{1,2}$. Tal multiplicidad de términos refleja que ninguno de ellos corresponde a la traducción idónea, hecho posiblemente explicado por carecer de una palabra en español para el término odds. Basándose en una serie de preguntas y ejemplos, el presente artículo explica los fundamentos matemáticos y metodológicos subyacentes al concepto de Odds Ratio, con el objetivo de facilitar su interpretación a clínicos e investigadores. Para profundizar más en este tema recomendamos la lectura del artículo de Bland et al. ${ }^{3}$ y los capítulos de Gordis ${ }^{4}$ y de Jaeschke et al. ${ }^{5}$.

\section{¿Qué es un Odds?}

Rutinariamente expresamos la posibilidad de ocurrencia de un evento de interés usando probabilidades. Por ejemplo, si el pronóstico del tiempo anuncia que la probabilidad de lluvia durante junio es $40 \%$, entendemos que es posible que llueva en 12/30 días que tiene dicho mes. Una forma alternativa de representar la posibilidad de ocurrencia de un evento de interés es mediante el uso de odds, definidos como un cociente entre el 
número de eventos y el número de "no eventos". Siguiendo el ejemplo, el odds de lluvia durante junio corresponde al cociente entre el número de días de lluvia y "no lluvia" de aquel mes, es decir, 12/18. Probabilidades y odds son intercambiables: una probabilidad puede convertirse en odds mediante la fórmula (probabilidad/(1-probabilidad)), y un odds convertirse en una probabilidad mediante la fórmula (odds/(odds+1)). La Tabla 1 ilustra la relación entre probabilidades y odds. A mayor probabilidad, mayor es la diferencia numérica entre ambos.

\section{¿Qué es un Odds Ratio?}

Supongamos que usted está a cargo de la organización de un paseo al aire libre, y duda si realizarlo en junio o noviembre. El pronóstico del tiempo anuncia que la probabilidad de lluvia en noviembre es $10 \%$ (es decir, es posible que llueva en 3/30 días que tiene dicho mes), siendo su odds de lluvia equivalente a $3 / 27$. ¿De qué manera compararía la posibilidad de que llueva en junio versus noviembre? La forma más simple es mediante el cálculo del Riesgo Relativo (RR). En el ejemplo, el RR corresponde al cociente entre la probabilidad de que llueva en junio dividido por la probabilidad de que llueva en noviembre, y equivale a $(12 / 30) /(3 / 30)=4,0$. Su interpretación es fácilmente comprensible: "la probabilidad de

Tabla 1. Equivalencia entre Riesgo y Odds

\begin{tabular}{|cc|}
\hline Riesgo & Odds \\
0,1 & $0,1 / 0,9=0,11$ \\
0,2 & $0,2 / 0,8=0,25$ \\
0,3 & $0,3 / 0,7=0,43$ \\
0,4 & $0,4 / 0,6=0,67$ \\
0,5 & $0,5 / 0,5=1,00$ \\
0,6 & $0,6 / 0,4=1,50$ \\
0,7 & $0,7 / 0,3=2,33$ \\
0,8 & $0,8 / 0,2=4,00$ \\
0,9 & $0,9 / 0,1=9,00$ \\
\hline
\end{tabular}

Riesgo $=($ odds $/($ odds +1$))$. Odds $=($ riesgo/(1-riesgo $))$. Los riesgos toman valores entre 0 y 1 , y los odds entre 0 e infinito. A mayor magnitud de riesgo, mayor es la diferencia numérica con su respectivo odds. Fuente: Elaboración propia. que llueva en junio es 4,0 veces la probabilidad de que llueva en noviembre" (nótese que el término "probabilidad" es intercambiable por "riesgo"). Análogamente, Odds Ratio (OR) se define como un cociente entre dos odds. En el ejemplo, el OR corresponde al odds de que llueva en junio dividido por el odds de que llueva en noviembre, y equivale a $(12 / 18) /(3 / 27)=6,0$. Su interpretación es un poco más compleja: "la razón de posibles días de lluvia versus "no lluvia" es 6 veces mayor en junio en comparación a noviembre". Notará el lector que tanto el valor del RR como del OR harán optar por organizar el paseo al aire libre durante el mes de noviembre, minimizando así la posibilidad de que la lluvia arruine dicha actividad.

\section{¿Cuándo utilizar Riesgo Relativo, y cuándo Odds Ratio?}

Numerosas investigaciones expresan sus resultados en forma de OR, en lugar de hacerlo de forma de RR. Pareciera ser que el reporte de OR en lugar de RR es muy común: en una serie de 151 estudios que reportaban OR como medida de efecto, Holcomb et al. encontraron que en $71 \%$ era factible calcular el RR, y en $26 \%$ se interpretaba el OR como RR, sin una clara justificación ${ }^{6}$. La decisión de la medida de efecto a utilizar se fundamenta en aspectos metodológicos y matemáticos, los cuales procedemos a detallar.

\section{Aspectos metodológicos}

Anteriormente definimos RR como un cociente entre dos probabilidades o dos riesgos, siendo el concepto de riesgo equivalente al concepto epidemiológico de "incidencia" (Nota: tasa de incidencia es el número de casos nuevos que se presentan en un período específico, dividido por el total de sujetos expuestos a transformarse en casos nuevos en igual período). La estimación de incidencia solamente es factible en estudios prospectivos, por consiguiente, el cálculo de RR se restringe exclusivamente a este tipo de estudios (ensayos controlados aleatorizados y estudios de cohorte). Los OR constituyen una medida de efecto alternativa al RR, siendo uno de sus méritos permitir expresar los resultados de estudios retrospectivos y transversales.

Surge entonces la pregunta: ¿por qué algunos estudios prospectivos, particularmente los de tipo 
observacional (estudios de cohorte) a menudo utilizan $\mathrm{OR}$ en vez del $\mathrm{RR}$, siendo este último más simple de calcular y fácil de interpretar? Ello ocurre debido a que en los estudios de cohorte los grupos a comparar no difieren únicamente en su condición de expuesto versus no-expuesto, sino también en la frecuencia con que se presentan en cada grupo otros factores de riesgo de ocurrencia del evento de interés, los cuales son responsables del llamado sesgo de confusión ${ }^{7}$. Existen diversas formas de minimizar el sesgo de confusión, siendo una estrategia estadística comúnmente utilizada los modelos de regresión logística. Estos modelos permiten calcular medidas de efecto ajustadas por variables confundentes (minimizando el sesgo de confusión), sin embargo, dichos modelos entregan como resultado OR ajustados, y no RR ajustados. Cabe destacar la existencia de otras estrategias de análisis estadístico que permiten el cálculo de RR ajustados por variables confundentes (por ejemplo, modelos de regresión log binomial), minimizando así el uso de OR en estudios de cohorte ${ }^{8}$.

\section{Aspectos matemáticos}

Comenzaremos asumiendo, en base a lo explicado en el punto anterior, que la disyuntiva entre utilizar OR versus RR se circunscribe a estudios prospectivos. OR y RR son intercambiables si se cumplen las siguientes condiciones: (i) el evento de interés ocurre de manera infrecuente en los grupos a comparar, y (ii) el tamaño del efecto es pequeño (es decir, el OR y el RR son cercanos a 1,00). Para un mismo estudio, el OR tiende a magnificar el tamaño del efecto, en comparación al RR. Sin embargo, cuando el evento de interés ocurre de manera infrecuente en los grupos a comparar, esta tendencia se minimiza ${ }^{5}$. Como regla general decimos que OR y RR son intercambiables cuando la frecuencia del evento de interés en la muestra total es igual o menor a 10\%, sin embargo, la decisión de intercambiar ambas medidas de efecto debe fundamentarse también en argumentos de tipo clínico (Figura 1).

Por otro lado, debemos reconocer un mérito del OR por sobre el RR: si se revierte el evento de interés en el análisis y se mira el evento favorable (ej. sobrevida) en vez del efecto desfavorable (ej. mortalidad), la asociación tendrá un OR recíproco, hecho que no ocurre para el RR. Considere el siguiente ejemplo: el riesgo de sanar en pacientes que consumen el fármaco A es 60/100, y el riesgo de sanar en pacientes que consumen el fármaco $\mathrm{B}$ es 20/100. El RR de sanar en pacientes que consumen el fármaco A versus B equivale a 3,0 y su OR equivale a 6,0 . Si se analiza el evento desfavorable, el riesgo de fallecer en pacientes que consumen el fármaco A es 40/100, y el riesgo de fallecer en pacientes que consumen el fármaco B es 80/100. Visto de esta manera, el RR de fallecer en pacientes que consumen el fármaco $\mathrm{A}$ versus $\mathrm{B}$ equivale a 0,5 (cifra que no es el recíproco de 3,0 ), y su OR equivale a 0,17 (cifra que sí es el recíproco de 6,0$)^{5}$.

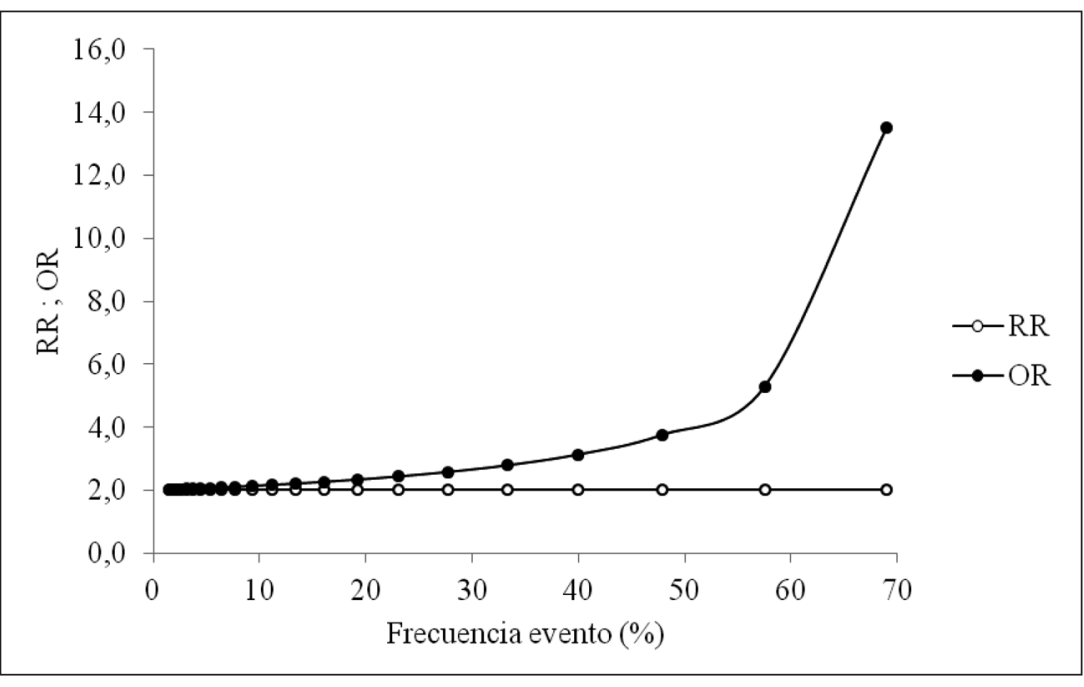

Figura 1. Comparación del RR y OR, según frecuencia del evento de interés en la muestra total. Valores de OR y RR calculados en una muestra constituida por 100 sujetos expuestos y 100 sujetos no expuestos, siendo la proporción de sujetos con el evento de interés en el grupo expuesto el doble de la observada en el grupo no expuesto $(R R=2,0)$. A medida que aumenta la frecuencia del evento de interés en la muestra total, el OR difiere más el RR, magnificando el tamaño del efecto. Fuente: Elaboración propia. 


\section{¿Cuáles son las diferencias en el cálculo e interpretación de OR en estudios prospectivos versus retrospectivos?}

Un estudio es temporalmente prospectivo cuando la muestra utilizada se conforma a partir de la exposición o intervención, observándose hacia el futuro la ocurrencia del evento de interés. Ejemplo de estudios prospectivos son los ensayos controlados aleatorizados y los estudios de cohor$\mathrm{te}^{9}$. Por el contrario, un estudio es temporalmente retrospectivo cuando la muestra utilizada se conforma a partir del evento de interés, indagándose en el pasado la presencia de una determinada exposición. Ejemplo de estudio retrospectivo son los estudios de casos y controles ${ }^{10}$. Los ejemplos 1 y 2, presentados al final de este artículo, resumen las fórmulas utilizadas para el cálculo de $\mathrm{OR}$ en estudios prospectivos: $\mathrm{OR}=(\mathrm{a} / \mathrm{b}) /(\mathrm{c} / \mathrm{d})$ (ejemplo $\left.\mathrm{n}^{\circ} 1\right)$ y retrospectivos: $\mathrm{OR}=(\mathrm{a} / \mathrm{c}) /(\mathrm{b} / \mathrm{d})$ (ejemplo $\mathrm{n}^{\circ} 2$ ). Nótese que ambas fórmulas son epidemiológicamente diferentes. La primera fórmula corresponde a un cociente entre el odds de presentar el evento de interés en los expuestos o intervenidos (a/b) y el odds de presentar el evento de interés en los "no expuestos" o "no intervenidos" (c/d). Por su parte, la segunda fórmula corresponde a un cociente entre el odds de exposición en los sujetos con el evento de interés $(\mathrm{a} / \mathrm{c})$ y el odds de exposición en los sujetos sin el evento de interés (b/d). Sin embargo, matemáticamente ambas fórmulas son equivalentes, $\mathrm{e}$ incluso pueden fusionarse en una sola: $\mathrm{OR}=(\mathrm{a} \times \mathrm{d}) /(\mathrm{c} \times \mathrm{d})$. No obstante ello, resulta altamente recomendable el uso de las fórmulas descritas para cada tipo de diseño en particular, pues ello ayuda a interpretar correctamente el $\mathrm{OR}$, respetando la temporalidad del diseño que originó los resultados. Hacemos notar que tanto la explicación recién expuesta como el ejemplo $\mathrm{n}^{\circ} 2$ corresponden a estudios de casos y controles "no pareados". En los estudios de casos y controles "pareados" por una o más variables confundentes el OR se calcula e interpreta de una manera distinta, excediendo su explicación el propósito del presente artículo.

\section{¿Cómo se calculan e interpretan OR en estudios transversales?}

En un estudio transversal la muestra es seleccionada sin conocer a priori la condición de cada sujeto respecto a la exposición y el evento de interés. Una vez seleccionada la muestra, se procede a determinar en cada sujeto su condición de expuesto versus no expuesto, y la presencia o ausencia del evento de interés, midiéndose ambas variables en forma simultánea ${ }^{11}$. Los estudios transversales no permiten el cálculo de tasas de incidencia, pues carecen de un sentido de observación prospectivo; por consiguiente, resulta metodológicamente incorrecto calcular RR como medida de efecto. En ellos, para expresar la frecuencia de ocurrencia de un evento de interés se utilizan los conceptos de "prevalencia" (número de sujetos que presentan el evento de interés en un momento o período específico, dividido por el total de sujetos expuestos a presentar dicho evento en igual momento o período) y de "odds de prevalencia" (cociente entre el número de sujetos que presentan el evento de interés y el número de sujetos que no lo presentan, en igual momento o período). Los conceptos de prevalencia y odds de prevalencia originan dos medidas de efecto: Razón de Prevalencia (RP) y Odds Ratio de Prevalencia (ORP). La razón de prevalencia corresponde a un cociente entre la prevalencia del evento de interés en el grupo expuesto versus no expuesto; por su parte, el ORP corresponde a un cociente entre el odds de prevalencia en el grupo expuesto versus no expuesto. Matemáticamente la RP de un estudio transversal se calcula de la misma forma que el RR de un estudio prospectivo: $(\mathrm{a} / \mathrm{a}+\mathrm{b}) /(\mathrm{c} / \mathrm{c}+\mathrm{d}), \mathrm{y}$ el ORP de un estudio transversal se calcula de la misma forma que el OR de un estudio prospectivo: $(\mathrm{a} / \mathrm{b}) /(\mathrm{c} / \mathrm{d})$ (ejemplo $\left.\mathrm{n}^{\circ} 3\right)$. Sin embargo, la interpretación de estas medidas de efecto es diferente, debido a que provienen de diseños distintos.

Surge la pregunta: ¿en un estudio transversal, debemos expresar los resultados como RP u ORP? ${ }^{12}$ Los argumentos que rigen la elección de RP versus ORP son los mismos que rigen la elección de RR versus OR (como fuera explicado en la pregunta $\mathrm{n}^{\circ} 3$ ). Por su parte, al igual que en los estudios de cohorte, es frecuente encontrar estudios transversales que reportan sus resultados en forma de ORP en lugar de hacerlo en forma de $\mathrm{RP}$, debido a que utilizan modelos de regresión logística como estrategia estadística para minimizar el sesgo de confusión. Tales modelos entregan sus resultados en forma de ORP ajustados por una o más variables confundentes.

Por último, es importante señalar que en al- 
gunos estudios transversales no es fácil establecer cuál es la exposición y cuál es el evento de interés, de modo que indistintamente pueden utilizarse las formulas ORP $=(\mathrm{a} / \mathrm{b}) /(\mathrm{c} / \mathrm{d})$, o bien ORP $=(\mathrm{a} / \mathrm{c}) /$ (b/d), ya que ambas fórmulas conducen al mismo resultado (como fuera explicado en la pregunta $n^{\circ}$ 4). Sin embargo, la lectura del ORP dependerá de qué variable se considere como exposición y qué variable se considere como evento de interés. Para ello recomendamos evaluar la secuencia lógica en que se presentan las variables en estudio, y así determinar cuál corresponde a la exposición y cuál corresponde al evento de interés.

\section{¿Qué información aporta el intervalo de confianza de un OR?}

En el ejemplo $n^{\circ} 1$, la asociación entre antecedente de migraña y síndrome hipertensivo del embarazo fue estudiada en base a una muestra de 685 embarazadas, siendo el $\mathrm{OR}=3,1$ y su intervalo de confianza 95\% 1,6-6,3. Ahora bien, dicho estudio podría haber utilizando una muestra diferente, de hecho, existen muchísimas posibles muestras de 685 embarazadas. Surge la pregunta: ¿cuál es el rango de valores que tomaría el OR calculado en múltiples muestras de 685 embarazadas? La respuesta a esta interrogante se obtiene a partir del intervalo de confianza del OR, medida de precisión que informa la variabilidad del OR calculado en un estudio. La interpretación del intervalo de confianza $95 \%$ calculado en el ejemplo $\mathrm{n}^{\circ} 1$ es la siguiente: si se repitiera el estudio múltiples veces, utilizando diferentes muestras de 685 embarazadas, 95\% de las muestras presentaría un OR entre 1,6 y 6,3. Ello nos conduce a concluir que el valor "real" (poblacional) del OR se encuentra entre dichos valores, con $95 \%$ de probabilidad. Esta medida de precisión tiene una interpretación práctica: los límites de un intervalo de confianza reflejan el tamaño de efecto mínimo $(\mathrm{OR}=1,6)$ y máximo $(\mathrm{OR}=6,3)$ asociado a una determinada exposición o intervención. La precisión del OR depende del tamaño muestral: para un mismo nivel de confianza, a mayor tamaño muestral más pequeño será el intervalo, por ende, más precisa será la estimación del OR "real" (poblacional).

La segunda información que aporta el intervalo de confianza de un OR tiene relación con su significación estadística. Imaginemos que a partir de una muestra de 100 sujetos se calcula un $\mathrm{OR}=$
1,4 , siendo su intervalo de confianza 95\% 0,5-3,7. Su interpretación es la siguiente: si se repitiera el estudio utilizando múltiples muestras de 100 sujetos, 95\% de ellas presentaría un OR entre 0,5 y 3,7 . Cuando un intervalo de confianza $95 \%$ incluye el valor del "no efecto" ( $O R=1,0)$, aceptamos que el OR "real" (poblacional) pudiese ser 1,0, por consiguiente, decimos que la asociación en estudio no es estadísticamente significativa (como ocurre en este ejemplo). Por el contrario, cuando un intervalo de confianza 95\% excluye el valor del "no efecto", descartamos que el OR "real" (poblacional) pudiese ser 1,0, por consiguiente, decimos que la asociación en estudio es estadísticamente signifcativa. No obstante lo anterior, es importante precisar un concepto: un OR estadísticamente significativo no necesariamente es biológica o clínicamente relevante, y viceversa.

En el ejemplo $n^{\circ} 1$, el intervalo de confianza 95\% del OR fue 1,6-6,3. Este resultado permite afirmar que el riesgo de SHE en las embarazadas con antecedente de migraña es significativamente mayor al de las embarazadas sin este antecedente. Una explicación más detallada sobre intervalos de confianza puede encontrarse en el capítulo de Guyatt et al. ${ }^{13}$.

\section{Conclusión}

Odds Ratio (OR) es una medida de efecto comúnmente utilizada para comunicar los resultados de una investigación en salud. Frecuentemente intercambiamos OR por RR, sin embargo, dicho intercambio se rige por una serie de consideraciones metodológicas, matemáticas y clínicas. Recomendamos al lector utilizar RR toda vez que sea metodológicamente posible, dada la simpleza de su cálculo y facilidad de interpretación, limitando el uso de OR a estudios retrospectivos y a circunstancias en las cuales se utilizan modelos de regresión logística como estrategia estadística para reducir el sesgo de confusión ${ }^{14}$. Asimismo, la interpretación de un OR debe realizarse respetando la temporalidad del estudio que le dio origen.

\section{Ejemplo $n^{\circ} 1$. Cálculo e interpretación de OR en estudios prospectivos (ensayos clínicos aleatorizados y estudios de cohorte)}

Se evaluó la asociación entre el antecedente de migraña y el desarrollo de síndrome hipertensivo 
del embarazo (SHE) utilizando un estudio de cohorte $^{15}$. Para ello siguieron a 685 embarazadas normotensas entre 11 y 16 semanas de gestación, 264 con antecedente de migraña y 421 sin este antecedente. En el grupo de embarazadas con antecedente de migraña 24 desarrollaron SHE y 240 no lo hicieron; por su parte, en el grupo de embarazadas sin antecedente de migraña 13 desarrollaron SHE y 408 no lo hicieron.

\begin{tabular}{|cccc|}
\hline \multicolumn{4}{c|}{$\begin{array}{c}\text { Evento de interés: } \\
\text { Síndrome hipertensivo } \\
\text { del embarazo (SHE) }\end{array}$} \\
$\begin{array}{l}\text { Exposición: } \\
\text { Antecedente } \\
\text { de migraña }\end{array}$ & $\begin{array}{l}\text { Ocurre } \\
\text { No ocurre }\end{array}$ & Total \\
Sí & 24 (a) & 240 (b) & 264 \\
No & 13 (c) & 408 (d) & 421 \\
\hline Total & 37 & 648 & 685 \\
\hline
\end{tabular}

RR de SHE en embarazadas expuestas versus no expuestas $=(\mathrm{a} / \mathrm{a}+\mathrm{b}) /(\mathrm{c} / \mathrm{c}+\mathrm{d})=(24 / 264) /$ $(13 / 421)=2,9($ IC95\% 1,5-5,7).

OR de SHE en embarazadas expuestas versus no expuestas $=(\mathrm{a} / \mathrm{b}) /(\mathrm{c} / \mathrm{d})=(24 / 240) /$ $(13 / 408)=3,1($ IC95\% 1,6-6,3) .

Interpretación OR: "la razón entre ocurrencia versus no ocurrencia de SHE es 3,1 veces mayor en embarazadas con antecedente de migraña en comparación a embarazadas sin este antecedente. Esta asociación es estadísticamente significativa”.

\section{Ejemplo $n^{\circ}$ 2. Cálculo e interpretación de OR en estudios retrospectivos (estudios de casos y controles no pareados)}

Se evaluó la magnitud de asociación entre hiperhomocisteinemia (HHC, definida como nivel de homocisteína mayor o igual a $15 \mu \mathrm{mol} / \mathrm{l}$ ) y cardiopatía isquémica crónica empleando un estudio de casos y controles no pareados ${ }^{16}$. La muestra estuvo constituida por 114 casos (sujetos con cardiopatía isquémica crónica) y 295 controles (sujetos sin cardiopatía isquémica crónica). Entre los casos, 33 sujetos presentaban HHC y 81 sujetos no la presentaban, mientras que entre los controles 38 sujetos presentaban HHC y 257 no la presentaban.

\begin{tabular}{|cccc|}
\hline \multicolumn{4}{c|}{$\begin{array}{c}\text { Evento de interés: } \\
\text { Cardiopatía isquémica } \\
\text { crónica }\end{array}$} \\
$\begin{array}{c}\text { Exposición: } \\
\text { Hiperhomo- } \\
\text { cisteinemia } \\
\text { (HHC) }\end{array}$ & $\begin{array}{c}\text { Presente } \\
\text { (Casos) }\end{array}$ & $\begin{array}{c}\text { Ausente } \\
\text { (Controles) }\end{array}$ & Total \\
\hline Sí & 33 (a) & $38(b)$ & 71 \\
No & 81 (c) & 257 (d) & 338 \\
\hline Total & 114 & 295 & 409 \\
\hline
\end{tabular}

OR de HHC en sujetos casos versus controles $=(\mathrm{a} / \mathrm{c}) /(\mathrm{b} / \mathrm{d})=(33 / 81) /(38 / 257)=2,8($ IC95\% $1,6-4,7)$

Interpretación OR: "la razón entre presencia de $\mathrm{HHC}$ versus ausencia de $\mathrm{HHC}$ es 2,8 veces mayor en los sujetos con cardiopatía isquémica crónica en comparación a los sujetos sin esta patología. Esta asociación es estadísticamente significativa”.

\section{Ejemplo $n^{\circ}$ 3. Cálculo e interpretación de OR en estudios transversales}

Se evaluó la asociación entre obesidad e hipertensión arterial en escolares chilenos, empleando un estudio transversal ${ }^{17}$. La muestra estuvo constituida por 2.980 escolares entre 6 y 14 años, de los cuales 622 eran obesos (162 hipertensos y 460 no hipertensos) y 1.593 eran eutróficos (142 hipertensos y 1.451 no hipertensos). El resto de los escolares $(n=765)$ presentaban otros categorías de diagnóstico nutricional (bajo peso y sobrepeso).

\begin{tabular}{|lccc|}
\hline \multicolumn{4}{|c|}{$\begin{array}{c}\text { Evento de interés: } \\
\text { Hipertensión arterial } \\
\text { (HTA) }\end{array}$} \\
$\begin{array}{l}\text { Exposición: } \\
\text { Estado } \\
\text { nutricional } \\
\text { Obesos }\end{array}$ & $\begin{array}{c}\text { Presente } \quad \text { Ausente } \\
\text { Total }\end{array}$ & \\
\hline Eutróficos & 162 (a) & 460 (b) & 622 \\
\hline Total & 142 (c) & 1.451 (d) & 1.593 \\
\hline
\end{tabular}

Razón de Prevalencia de HTA en escolares obesos versus eutróficos $=(\mathrm{a} / \mathrm{a}+\mathrm{b}) /(\mathrm{c} / \mathrm{c}+\mathrm{d})=(162 / 622) /$ $(142 / 1.593)=2,9($ IC95\% 2,4-3,6)

OR de prevalencia de HTA en escolares obesos versus eutróficos $=(162 / 460) /(142 / 1.451)=3,6$ (IC95\% 2,8-4,6)

Interpretación OR de prevalencia: "la razón 
entre escolares hipertensos versus no hipertensos es 3,6 veces mayor en escolares obesos en comparación a escolares eutróficos. Esta asociación es estadísticamente significativa”.

\section{Referencias}

1. Martín-Moreno JM, Banegas JR. Sobre la traducción del término inglés odds ratio como oportunidad relativa. Salud Pública Mex 1997; 39: 72-4.

2. Rivera S, Larrondo FJ, Ortega JP. Evaluación de los resultados de un artículo sobre tratamiento. Rev Med Chile 2005; 133: 593-6.

3. Bland JM, Altman DG. Statistical notes. The odds ratio. BMJ 2000: 320: 1468.

4. Gordis L. Chapter 11. Estimating risk: is there an association? En: Gordis L. Epidemiology, Fourth Edition. Saunders; 2009. p. 201-14.

5. Jaeschke R, Walter S, El Barbary M, Guyatt G. Chapter 10.2. Understanding the results: more about odds ratios. En: Guyatt G, Rennie D, Meade M, Cook D. Users' Guides to the Medical Literature, Second Edition. McGrawHill; 2008. p. 221-9.

6. Holcomb WL, Chaiworapongsa T, Luke DA, Burgdorf KD. An odd measure of risk: use and misuse of the odds ratio. Obstet Gynecol 2001; 98: 685-8.

7. Grimes D, Schulz K. Bias and causal associations in observational research. Lancet 2002; 359: 248-52.

8. Mcnutt LA, Wu C, Xue X, Hafner JP. Estimating the relative risk in cohort studies and clinical trials of common outcomes. Am J Epidemiol 2003; 157: 940-3.

9. Grimes D, Schulz K. Cohort studies: marching towards outcomes. Lancet 2002; 359: 341-5.

10. Schulz K, Grimes DA. Case-control studies: research in reverse. Lancet 2002; 359: 431-4.

11. Hernández B, Velasco-Mondragón HE. Encuestas transversales. Salud Pública Mex 2000; 42: 447-55.

12. Zocchetti C, Consonni D, Bertazzi P. Relationship between prevalence rate ratios and odds ratios in crosssectional studies. Int J Epidemiol 1997; 26: 20-3.

13. Guyatt G, Walter S, Cook D, Wyer P, Jaeschke R. Chapter 8. Confidence intervals. En: Guyatt G, Rennie D, Meade M, Cook D. Users' Guides to the Medical Literature, Second Edition. McGraw-Hill; 2008. p. 99-108.

14. Deeks J. When can odds ratios mislead? Odds ratios should be used only in case-control studies and logistic regression analyses. BMJ 1998; 317: 1155-6.

15. Facchinetti F, Allais G, Nappi RE, D'amico R, Marozio L, Bertozzi L, ET AL. Migraine is a risk factor for hypertensive disorders in pregnancy: a prospective cohort study. Cephalalgia 2009; 29: 286-92.

16. Gallego-Luis R, Ruiz-García A, Gordillo-López FJ, DíazPuente MV, Esteban J, Gil de Miguel A. Estudio de casos y controles no emparejados: hiperhomocisteinemia y cardiopatía isquémica crónica. Aten Primaria 2006; 37: 325-31.

17. Bancalari R, Díaz C, Martínez-Aguayo A, Aglony M, Zamorano J, Cerda V, et al. Prevalencia de hipertensión arterial y su asociación con la obesidad en edad pediátrica. Rev Med Chile 2011; 139: 872-9. 